\title{
Semi-Finite Noncommutative Geometry and some Applications
}

\author{
A.L. Carey \\ J. Phillips \\ A. Rennie
}

Vienna, Preprint ESI 2061 (2008)

October 28, 2008

Supported by the Austrian Federal Ministry of Education, Science and Culture Available via http://www.esi.ac.at 


\title{
SEMI-FINITE NONCOMMUTATIVE GEOMETRY AND SOME APPLICATIONS
}

\author{
A.L. Carey* ${ }^{*}$ J. Phillips ${ }^{\sharp}$, A. Rennie* \\ * Mathematical Sciences Institute, \\ Australian National University, Canberra, ACT, AUSTRALIA \\ $\sharp$ Department of Mathematics and Statistics \\ University of Victoria, Victoria, BC, CANADA \\ acarey@maths.anu.edu.au, phillips@math.uvic.ca,adam.rennie@maths.anu.edu.au
}

\section{INTRODUCTION}

These notes are a summary of talks given in Shonan, Japan in February 2008 with modifications from a later series of talks at the Hausdorff Institute for Mathematics in Bonn in July 2008 and at the Erwin Schrödinger Institute in October 2008. The intention is to give a short discussion of recent results in noncommutative geometry (NCG) where one extends the usual point of view of [22] by replacing the bounded operators $B(\mathcal{H})$ on a Hilbert space $\mathcal{H}$ by certain sub-algebras; namely semi-finite von Neumann algebras. These are weakly closed *-subalgebras of the bounded operators on a Hilbert space that admit a faithful, normal semi-finite trace. The exposition is partly historical and intended to explain where this idea came from and how it links to other developments in index theory and NCG going back over 20 years.

For the authors a major motivating example of this so-called semi-finite NCG is the notion of spectral flow for paths of operators in a semi-finite von Neumann algebra. In these notes we will therefore focus on this example. The theory of analytic spectral flow as a whole is surveyed in some detail in the article [5] and so it would be superfluous to repeat that discussion here. We will summarise in Section 2 the details needed for this current discussion, especially those relevant to Section 4 . We mention that the classical topological theory of spectral flow is described in many places starting with $[1,2]$ and in textbook form in $[8,42]$. The analytic theory is a more recent development and really starts with [48] although in the standard $B(\mathcal{H})$ case there is overlap between [42] and [48]. We will not in these notes address the related notion of 'higher spectral flow' [31] or the extensive literature built on that. The idea of spectral flow as an intersection number does not apply in the semi-finite theory. It was Mathai in 1992, [41], who asked the question about whether there is a semi-finite spectral flow in connection with

Atiyah's $L^{2}$-index theorem. Motivated by a fundamental paper of Getzler 
in 1993, [33], J. Phillips, [48, 49], independently studied analytic approaches to spectral flow in von Neumann algebras (see also [46, 47] for an early attempt). We will summarise this point of view below referring to [5] for more information. It is now appreciated that the natural setting for spectral flow is as an invariant associated to paths of operators in a semi-finite von Neumann algebra.

We will deal only with separable spaces $\mathcal{H}$. A large part of our discussion reduces to familiar theory when $\mathcal{N}=B(\mathcal{H})$ equipped with its standard trace (this is the situation in Alain Connes' book [22]). The point is, however, that the semi-finite NCG theory is not just an extension of what is known: it requires new ideas and these feed back into the standard $\mathcal{N}=B(\mathcal{H})$ case producing refinements and advances there as well.

Semi-finite NCG needs an extension of the theory of Fredholm operators in the sense of Breuer, [9]. Breuer was the first to tackle the problem of how to modify the $B(\mathcal{H})$-Fredholm theory to handle semi-finite algebras. For the purposes of the current article we will use the following consequence of Breuer's theory [50]. If $\mathcal{N}$ is a semifinite von Neumann algebra and $\tau$ is a fixed faithful normal semifinite trace then to say that $F \in \mathcal{N}$ is $\tau$-Fredholm is shorthand for saying that $F$ is invertible in the $\tau$-Calkin algebra (semi-finite Atkinson's theorem). This latter algebra is the quotient of $\mathcal{N}$ by the norm closed ideal $\mathcal{K}_{\tau}$ generated by the $\tau$-finite projections. A careful discussion of what is needed in the way of an extension of Breuer's work for NCG purposes is contained in [16]. In these notes we will also study unbounded Fredholm operators. While there are several ways to do this we will only use the viewpoint of the Riesz map on unbounded operators. That is, if $D$ is unbounded we will consider the map $D \rightarrow F_{D}:=D\left(1+D^{*} D\right)^{-1 / 2}$ and say that the unbounded operator $D$ is $\tau$-Fredholm if its bounded image $F_{D}$ is $\tau$-Fredholm.

Index theory provides a major motivation for NCG and it led to the notion of spectral triple as a result of the work of Kasparov, [38]. We will study odd index theory and the associated problem of calculating spectral flow in the general framework of spectral triples, formerly known as unbounded Fredholm modules.

Recall the following fundamental definition from [22].

Definition A spectral triple $(\mathcal{A}, \mathcal{H}, \mathcal{D})$ consists of a (unital) *-subalgebra $\mathcal{A}$ of the bounded operators on a separable Hilbert space $\mathcal{H}$ and an unbounded self-adjoint operator $\mathcal{D}: \operatorname{dom} \mathcal{D} \subset \mathcal{H} \rightarrow \mathcal{H}$ with compact resolvent such that $[\mathcal{D}, a]$ extends to a bounded operator for all $a \in \mathcal{A}$

The spectral triple is even if there is a self-adjoint bounded involution $\Gamma$ which anticommutes with $\mathcal{D}$ and commutes with $\mathcal{A}$; otherwise it is odd. 
The key axiom here is the compact resolvent condition. This tells us that $\mathcal{D}$ is an (unbounded) Fredholm operator, a condition natural from the viewpoint of index theory for elliptic operators on compact manifolds. However there are situations where this compactness assumption fails. One such situation is Atiyah's $L^{2}$-index theorem where one has elliptic operators acting on sections of bundles on the universal cover of a compact manifold. These elliptic operators have a resolvent which is in the ideal of compact operators in a von Neumann algebra $\mathcal{N}$ which is not $\mathcal{B}(\mathcal{H})$ and they are not compact in the usual sense. This is exactly the setting of Mathai's question of whether the $L^{2}$-index theorem for odd dimensional manifolds could be thought of as calculating a kind of spectral flow and it is naturally answered by semi-finite NCG (see [5]).

Briefly then, semi-finite NCG uses the framework of spectral triples, but replaces the bounded operators on a Hilbert space and their ideal of compact operators by a general semi-finite von Neumann algebra and its corresponding ideal of compacts. To work in this generality one needs a theory of operator ideals in semi-finite von Neumann algebras. This was provided in a fundamental paper of T. Fack and H. Kosaki, [32]. Fortunately the theory needed goes through, in particular the definition of the 'Dixmier ideal' $\mathcal{L}^{(1, \infty)}$ and the Wodzicki residue (see $[18,6]$ ). A survey of all the refinements of the Fack-Kosaki work that semi-finite NCG requires is contained in [21] and we will not go over this ground again here.

A second motivation for studying semi-finite NCG is the question of finding analytic formulas for spectral flow. The first such general formula is due to Getzler [33] although his arguments rely on the topological definition of spectral flow as an intersection number. It is by no means clear that the notion of an intersection number can be used in the case where the operators in question have continuous spectrum as occurs in the semi-finite theory. Thus new ideas are needed. The paper [11] initiated the development of a semi-finite theory. It introduced the notion of semi-finite spectral triple and many of the other techniques needed to produce formulas to calculate spectral flow in the semi-finite setting.

The next development was in the form of a preliminary version of the manuscript of Benameur-Fack [6]. Motivated by foliations they introduced both odd and even versions of semi-finite NCG and their work provided an impetus for the present authors to press ahead with a new proof of the semi-finite local index formula. In fact, it wasn't until the existence in the semi-finite case of all these examples, such as the $L^{2}$-index theorem of Atiyah, foliations and analytic spectral flow, that a general theory seemed to be worthwhile.

A further ingredient comes from early work in the 1980s of which we only became aware recently. This is contained in the paper [23] and is perhaps the earliest indication of the connection between semi-finite Kasparov theory 
and cyclic cohomology. It provides an additional motivation for semi-finite NCG. In [23], Connes and Cuntz show that cyclic $n$-cocycles for an appropriate algebra $A$ are in one-to-one correspondence with traces on a certain ideal $J^{n}$ in the free product $A * A$. Assuming some positivity for this trace yields the same kind of 'semi-finite Kasparov modules' as are described in [37]. In other words, to realise all the cyclic cocycles for an algebra will, in general, necessitate considering semifinite Fredholm modules and semi-finite spectral triples.

In these notes we will sketch the key notions and some of the main results that have been established by the current authors or their collaborators in the last decade. We start with our NCG approach [18] to an index theorem of Lesch [39] for Toeplitz operators with noncommutative symbol. This can be seen as a special case of the local index formula in semi-finite NCG as we will explain in later Sections. Lesch's result starts with a symbol algebra admitting a finite trace. It motivated us to look for an analogous result when we start with a KMS state on the symbol algebra. We started to investigate this in the early 2000's through the study of a range of examples such as the Cuntz algebras $O_{n}$ and quantum $S U(2)$. These examples forced us to consider an adaptation of semifinite NCG to settings where more general von Neumann algebras play a role, specifically type III von Neumann algebras and to replace traces with KMS states and weights. As we will explain this led naturally to index theorems in twisted cyclic theory and to the consideration of equivariant $K$-theory.

An unrelated approach to the type III setting has been proposed by ConnesMoscovici, [27]. In their setting they retain the trace and the usual pairing with $K$-theory by modifying the construction/definition of spectral triples to handle the 'twist by an automorphism' (this 'twist' is what leads to twisted cyclic theory).

Acknowledgements. ALC thanks Keio University for its hospitality in supporting his visit to Japan and the Erwin Schrödinger Institute for providing an opportunity to polish these notes. All authors thank the Hausdorff Institute for Mathematics for support. ALC and AR acknowledge the support of the Australian Research Council and JP acknowledges the support of NSERC.

\section{Spectral Flow}

2.1. Definitions. We recall some basic notions. A trace on a von Neumann algebra $\mathcal{N}$ is a map $\tau$ on the positive elements $\mathcal{N}^{+}$such that

(i) $\tau(a) \in \mathbf{R}^{+} \cup\{\infty\}$ for all $a \in \mathcal{N}^{+}$,

(ii) $\tau(a+b)=\tau(a)+\tau(b), \tau(\alpha a)=\alpha \tau(a)$, for all $a, b \in \mathcal{N}^{+}$and $\alpha>0$ in 
$\mathbf{R}^{+}$

(iii) $\tau\left(u a u^{*}\right)=\tau(a)$ for all $a \in \mathcal{N}^{+}$and unitaries $u \in \mathcal{N}$.

We may extend $\tau$ linearly to $\mathcal{N}$. Faithful means $\tau(a)=0$ for $a \in \mathcal{N}^{+}$if and only if $a=0$. Normal (which is equivalent to weakly lower semicontinuous) means that if there is a net $\left\{a_{\lambda}\right\} \in \mathcal{N}^{+}$with supremum $a \in \mathcal{N}^{+}$then $\tau(a)=\sup _{\lambda} \tau\left(a_{\lambda}\right)$. Semifinite means that $\tau(a)$ is the supremum of $\tau(b)$ for $b<a$ and $a, b \in \mathcal{N}^{+}$with $\tau(b)<\infty$.

Next we summarise some of $[48,49,5]$. Let $\mathcal{X}_{0}:=\mathcal{X}_{[0, \infty)}$ be the characteristic function of the interval $[0, \infty)$ so that if $T$ is any self-adjoint operator in a von Neumann algebra $\mathcal{N}$ then $\mathcal{X}_{0}(T)$ is a projection in $\mathcal{N}$. Let $\pi$ be the quotient map onto the $\tau$-Calkin algebra of $\mathcal{N}$.

Definition. Let $\mathcal{N}$ be a semifinite von Neumann algebra with fixed semifinite, faithful, normal trace, $\tau$. Let $\mathcal{F}^{s a}$ denote the space of all self-adjoint $\tau$-Fredholm operators in $\mathcal{N}$. Let $\left\{F_{t}\right\}$ be any continuous path in $\mathcal{F}^{s a}$ (indexed by some interval $[a, b])$. Then $\left\{\mathcal{X}_{0}\left(F_{t}\right)\right\}$ is a (generally discontinuous) path of projections in $\mathcal{N}$. But $\pi\left(\mathcal{X}_{0}\left(F_{t}\right)\right)=\mathcal{X}_{0}\left(\pi\left(F_{t}\right)\right)$ and since the spectra of $\pi\left(F_{t}\right)$ are bounded away from 0 , this latter path is continuous. By compactness we can choose a partition $a=t_{0}<t_{1}<\cdots<t_{k}=b$ so that for each $i=1,2, \cdots, k$

$$
\left\|\pi\left(\mathcal{X}_{0}\left(F_{t}\right)\right)-\pi\left(\mathcal{X}_{0}\left(F_{s}\right)\right)\right\|<\frac{1}{2} \quad \text { for all } t, s \text { in }\left[t_{i-1}, t_{i}\right] .
$$

Letting $P_{i}=\mathcal{X}_{0}\left(F_{t_{i}}\right)$ for $i=0,1, \cdots, k$ we define the spectral flow of the path $\left\{F_{t}\right\}$ to be the number:

$$
s f\left(\left\{F_{t}\right\}\right)=\sum_{i=1}^{k} e c\left(P_{i-1}, P_{i}\right) .
$$

Here $e c\left(P_{i-1}, P_{i}\right)$ is the essential co-dimension of the pair $P_{i-1}, P_{i}$ that is, the index of $P_{i-1} P_{i}$ as an operator from $P_{i} \mathcal{H}$ to $P_{i-1} \mathcal{H}$. Thus if we use the notation $Q_{F}$ to denote the kernel projection of a $\tau$-Fredholm operator $F$ then the essential codimension of the pair $P_{i-1}, P_{i}$ is $\tau\left(Q_{P_{i-1} P_{i}}\right)-\tau\left(Q_{P_{i} P_{i-1}}\right)$.

2.2. The spectral flow problem in spectral triples. In practice we need to handle unbounded operators. Let $\mathcal{D}$ be an unbounded self-adjoint operator on the Hilbert space $\mathcal{H}$, with $\mathcal{D}$ having $\tau$-compact resolvent in a semifinite von Neumann algebra $\mathcal{N}$.

Recalling the definition of spectral triple, we see that in NCG the following situation arises. We have $u$, a unitary in $\mathcal{N}$, such that $[\mathcal{D}, u]$ extends to a bounded operator. Then we want to study spectral flow along the path

$$
t \rightarrow \mathcal{D}+t u\left[\mathcal{D}, u^{*}\right], \quad t \in[0,1]
$$


The natural setting for this study is given in the following:

Definition. A semi-finite spectral triple $(\mathcal{A}, \mathcal{H}, \mathcal{D})$ consists of a (unital) *subalgebra $\mathcal{A}$ of a semi-finite von Neumann algebra $\mathcal{N}$ acting on a separable Hilbert space $\mathcal{H}$ and an unbounded self-adjoint operator $\mathcal{D}: \operatorname{dom} \mathcal{D} \subset \mathcal{H} \rightarrow$ $\mathcal{H}$ with $\tau$-compact resolvent in $\mathcal{N}$ such that $[\mathcal{D}, a]$ extends to a bounded operator for all $a \in \mathcal{A}$.

A problem which arises inevitably in the semi-finite case is that $\mathcal{D}$ can have zero in its continuous spectrum. Thus $|\mathcal{D}|^{-1}$ is typically unbounded. The solution to this problem is that whenever $|\mathcal{D}|^{-1}$ appears in the $B(\mathcal{H})$ case it is to be replaced with $\left(1+\mathcal{D}^{2}\right)^{-1 / 2}$ for the semi-finite case.

Semifinite spectral triples provide information that is different from that given by ordinary spectral triples. This is explained by a result of Kaad-NestRennie [37]. They show that a semifinite spectral triple for $\mathcal{A}$ represents an element of $K K^{1}(\mathcal{A}, J)$, where $J$ is the $\sigma$-unital norm closed ideal of compact operators in $\mathcal{N}$ generated by the resolvent of $\mathcal{D}$ and the commutators $\left[F_{\mathcal{D}}, a\right]$ for $a \in \mathcal{A}$. (Recall $F_{\mathcal{D}}=\mathcal{D}\left(1+\mathcal{D}^{2}\right)^{-1 / 2}$.)

Returning now to the question of spectral flow along the path

$$
\mathcal{D}_{t}^{u}:=(1-t) \mathcal{D}+t u \mathcal{D} u^{*}=\mathcal{D}+t u\left[\mathcal{D}, u^{*}\right]
$$

we first observe that this is a "continuous" path of unbounded self-adjoint $\tau$-Fredholm operators in the following sense. By Theorem A.8 of [11] the path,

$$
F_{t}^{u}:=\mathcal{D}_{t}^{u}\left(1+\left(\mathcal{D}_{t}^{u}\right)^{2}\right)^{-1 / 2}
$$

is a norm-continuous path of (bounded) self-adjoint $\tau$-Fredholm operators. Now we appeal to Phillips definition of spectral flow along this path $\left\{F_{t}^{u}\right\}$ and use it to define spectral flow along $\left\{\mathcal{D}_{t}^{u}\right\}$ by

$$
s f\left(\left\{\mathcal{D}_{t}^{u}\right\}\right):=s f\left(\left\{F_{t}^{u}\right\}\right) .
$$

This definition then recovers the pairing of the semi-finite K-homology class $[\mathcal{D}]$ with the K-theory class $[u] \in K_{1}(\mathcal{A})$.

The spectral flow for the path $\left\{\mathcal{D}_{t}^{u}\right\}$ is the essential co-dimension of two projections as follows. Let $\tilde{F}_{j}^{u}$ be the phase of $\mathcal{D}_{j}^{u}$ (which is the phase of $\left.F_{j}^{u}\right)$ for $j=1,2$. Thus $\tilde{F}_{j}^{u}=2 \mathcal{X}_{0}\left(\mathcal{D}_{j}\right)-1$. Now we introduce the path $\left\{\tilde{F}_{t}^{u}\right\}$ where $\tilde{F}_{t}^{u}=(1-t) \tilde{F}_{0}^{u}+t \tilde{F}_{1}^{u}$. For brevity write $\tilde{F}_{0}^{u}=2 P-1$ then $P^{\prime}=u P u^{*}$ satisfies $\tilde{F}_{1}^{u}=2 P^{\prime}-1$. Then we may show that $P P^{\prime}: P^{\prime} \mathcal{H} \rightarrow P \mathcal{H}$ is $\tau$-Fredholm and hence Phillips' definition gives

$$
s f\left(\mathcal{D}, u \mathcal{D} u^{*}\right):=e c\left(P, P^{\prime}\right):=\operatorname{Index}\left(P P^{\prime}\right)
$$


where $P^{\prime}=u P u^{*}$. This may also be written in terms of the index of generalised Toeplitz operators because

$$
\begin{aligned}
& \text { Index }\left(P P^{\prime}\right)=\text { Index }(P u P) \\
& \quad=\tau\left(Q_{P u P}\right)-\tau\left(Q_{P u^{*} P}\right) .
\end{aligned}
$$

Remark. Spectral flow is not interesting in the case of even semifinite spectral triples. This is because the existence of a grading operator $\Gamma \in \mathcal{N}$ commuting with $\mathcal{A}$ and anticommuting with $\mathcal{D}$ forces spectral flow to be zero. This is easy to check from the relations $\Gamma P \Gamma=1-P, \Gamma P^{\prime} \Gamma=1-P^{\prime}$ which tell us that

$$
\operatorname{Index}\left(P P^{\prime}\right)=\operatorname{Index}\left(\Gamma P P^{\prime} \Gamma\right)=\operatorname{Index}\left(P^{\prime} P\right)
$$

so that $\operatorname{Index}\left(P P^{\prime}\right)=0$.

2.3. Spectral flow formulas. There are formulas for this semi-finite spectral flow along $\left\{D_{t}^{u}\right\}$ which are best stated in a more general form where the endpoints are not unitarily equivalent. We change notation in this subsection to accommodate our later examples.

Let $\mathcal{D}_{a}$ be a self-adjoint densely defined unbounded operator on the Hilbert space $\mathcal{H}$, affiliated to the semifinite von Neumann algebra $\mathcal{M}$. We let $\mathcal{L}^{p}(\mathcal{M}, \phi)$ be the ideal of compact operators generated by positive operators whose $p^{t h}$ power is $\phi$-trace class for some fixed faithful, normal, semi-finite trace $\phi$ on $\mathcal{M}$. We assume that

$$
\left(1+\mathcal{D}_{a}^{2}\right)^{-1 / 2} \in \mathcal{L}^{p}(\mathcal{M}, \phi) .
$$

Let $\mathcal{D}_{b}$ differ from $\mathcal{D}_{a}$ by a bounded self adjoint operator $A$ in $\mathcal{M}$. We take any path $A_{t}$ in the self adjoint bounded operators that is piecewise $C^{1}$ in the uniform norm with $A_{a}=0$ and $A_{b}=A$ and then $\left\{\mathcal{D}_{t}=\mathcal{D}_{a}+A_{t}\right\} ; t \in[a, b]$ joins $\mathcal{D}_{a}$ and $\mathcal{D}_{b}$.

We will not go into detail here on the derivation of formulas to compute the spectral flow referring instead to the discussion in [12] and [14]. The formula that we will need later for the spectral flow along $\left\{\mathcal{D}_{t}\right\}$ is:

$$
\frac{1}{C_{r}} \int_{a}^{b} \phi\left(\dot{\mathcal{D}}_{t}\left(1+\mathcal{D}_{t}^{2}\right)^{-r}\right) d t+\frac{1}{2 C_{r}}\left(\eta_{\mathcal{D}_{b}}(r)-\eta_{\mathcal{D}_{a}}(r)\right)+\frac{1}{2}\left(\phi\left(P_{\operatorname{ker}} \mathcal{D}_{b}\right)-\phi\left(P_{\operatorname{ker} \mathcal{D}_{a}}\right)\right),
$$

where $\Re(r)>p / 2+1 / 2$,

$$
C_{r}=\frac{\Gamma(r)}{\sqrt{\pi} \Gamma(r-1 / 2)}
$$

and

$$
\eta_{\mathcal{D}}(r)=\int_{1}^{\infty} s^{-1 / 2} \phi\left(\mathcal{D}\left(1+s \mathcal{D}^{2}\right)^{-r}\right) d s
$$


This formula may be thought of as the integral of a one form on the affine space of bounded perturbations of $\mathcal{D}_{a}$. In addition the truncated $\eta$ and kernel terms arise from the endpoints and cancel when the endpoints are unitarily equivalent via a unitary in $\mathcal{M}$.

2.4. Lesch's Index Theorem. A motivating example of an index theorem that leads naturally to semi-finite NCG is a theorem of Matthias Lesch [40]. Although the original proof did not involve spectral triples it was discovered in [18] that $\left(^{*}\right)$ may be used to recover Lesch's result. The method by which this was done also showed that $(*)$ is related to the expression for spectral flow given in the fundamental paper of Connes-Moscovici [25] by the local index formula in NCG. Before moving to a discussion of developments motivated by [25] we remark that there has been a lot of work subsequent to the original papers [33] and [11] on spectral flow formulas. We mention, for example, a different approach using the so-called gap topology on unbounded Fredholm operators (when $\mathcal{M}=B(\mathcal{H})$ ) which appeared in [7]. Spectral flow formulae based on this approach in the semi-finite case were provided in [53] and the connection with Phillips ideas in the semi-finite von Neumann case clarified. Very recently a general expression for spectral flow as an integral of one forms on certain Banach submanifolds of the manifold of all self adjoint $\tau$-Fredholm operators was found in [19] to which we also refer for a more comprehensive set of references to research on this problem.

Now we explain the motivating example from [40]. The starting point is to consider a unital $C^{*}$-algebra $A$ with a faithful finite trace, $\tau$ satisfying $\tau(1)=1$ and a continuous action $\alpha$ of $\mathbf{R}$ on $A$ leaving $\tau$ invariant. We let $H_{\tau}$ denote the Hilbert space completion of $A$ in the inner product $(a \mid b)=\tau\left(b^{*} a\right)$. Then $A$ is a Hilbert algebra and the left regular representation of $A$ on itself extends by continuity to a representation, $a \mapsto \pi_{\tau}(a)$ of $A$ on $H_{\tau}$. In what follows, we will drop the notation $\pi_{\tau}$ and just denote the action of $A$ on $H_{\tau}$ by juxtaposition. We use the notation $U(B)$ to denote the unitary elements of a $*$-algebra $B$.

We now look at the induced representation, $\tilde{\pi}$, of the crossed product $C^{*}$ algebra $A \times{ }_{\alpha} \mathbf{R}$ on $L^{2}\left(\mathbf{R}, H_{\tau}\right)$. That is, $\tilde{\pi}$ is the representation $\pi \times \lambda$ obtained from the covariant pair, $(\pi, \lambda)$ of representations of the system $(A, \mathbf{R}, \alpha)$ defined for $a \in A, t, s \in \mathbf{R}$ and $\xi \in L^{2}\left(\mathbf{R}, H_{\tau}\right)$ by:

$$
(\pi(a) \xi)(s)=\alpha_{s}^{-1}(a) \xi(s)
$$

and

$$
\lambda_{t}(\xi)(s)=\xi(s-t)
$$


Then, for a function $x \in L^{1}(\mathbf{R}, A) \subset A \times{ }_{\alpha} \mathbf{R}$ the action of $\tilde{\pi}(x)$ on a vector $\xi$ in $L^{2}\left(\mathbf{R}, H_{\tau}\right)$ is defined as follows:

$$
(\tilde{\pi}(x) \xi)(s)=\int_{-\infty}^{\infty} \alpha_{s}^{-1}(x(t)) \xi(s-t) d t
$$

Now the twisted convolution algebra $L^{1}(\mathbf{R}, A) \cap L^{2}\left(\mathbf{R}, H_{\tau}\right)$ is a dense subspace of $L^{2}\left(\mathbf{R}, H_{\tau}\right)$ and also a Hilbert algebra in the given inner product. As such, there is a canonical faithful, normal, semifinite trace, $\operatorname{Tr}$, on the von Neumann algebra that it generates. Of course, this von Neumann algebra is identical with

$$
\mathcal{N}=\left(\tilde{\pi}\left(A \times_{\alpha} \mathbf{R}\right)\right)^{\prime \prime}
$$

For functions $x, y: \mathbf{R} \rightarrow A \subset H_{\tau}$ which are in $L^{2}\left(\mathbf{R}, H_{\tau}\right)$ and whose twisted left convolutions $\tilde{\pi}(x), \tilde{\pi}(y)$ define bounded operators on $L^{2}\left(\mathbf{R}, H_{\tau}\right)$, this trace is given by:

$$
\operatorname{Tr}\left(\tilde{\pi}(y)^{*} \tilde{\pi}(x)\right)=\langle x \mid y\rangle=\int_{-\infty}^{\infty} \tau\left(x(t) y(t)^{*}\right) d t
$$

In particular, if we identify $L^{2}(\mathbf{R})=L^{2}(\mathbf{R}) \otimes 1_{A} \subset L^{2}\left(\mathbf{R}, H_{\tau}\right)$ then any scalar-valued function $x$ on $\mathbf{R}$ which is the Fourier transform $x=\widehat{f}$ of a bounded $L^{2}$ function, $f$ will have the properties that $x \in L^{2}\left(\mathbf{R}, H_{\tau}\right)$ and $\tilde{\pi}(x)$ is a bounded operator. For such scalar functions $x$, the operator $\tilde{\pi}(x)$ is just the usual convolution by the function $x$ and is usually denoted by $\lambda(x)$ since it is just the integrated form of $\lambda$. The next Lemma follows easily from these considerations.

Lemma 2.1. With the hypotheses and notation discussed above

(i) if $h \in L^{2}(\mathbf{R})$ with $\lambda(h)$ bounded and $a \in A$, then defining $f: \mathbf{R} \rightarrow H_{\tau}$ via $f(t)=a h(t)$ we see that $f \in L^{2}\left(\mathbf{R}, H_{\tau}\right)$ and $\tilde{\pi}(f)=\pi(a) \lambda(h)$ is bounded,

(ii) if $g \in L^{1}(\mathbf{R}) \cap L^{\infty}(\mathbf{R})$ and $a \in A$ then $\pi(a) \lambda(\hat{g})$ is trace-class in $\mathcal{N}$ and

$$
\operatorname{Tr}(\pi(a) \lambda(\hat{g}))=\tau(a) \int_{-\infty}^{\infty} g(t) d t .
$$

For each $t \in \mathbf{R}, \lambda_{t}$ is a unitary in $U(\mathcal{N})$. In fact the one-parameter unitary group $\left\{\lambda_{t} \mid t \in \mathbf{R}\right\}$ can be written $\lambda_{t}=e^{i t \mathcal{D}}$ where $\mathcal{D}$ is the unbounded self-adjoint operator

$$
\mathcal{D}=\frac{1}{2 \pi i} \frac{d}{d s}
$$

which is affiliated with $\mathcal{N}$. In the Fourier Transform picture (i.e., the spectral picture for $\mathcal{D}$ ) of the previous proposition, $\mathcal{D}$ becomes multiplication by the 
independent variable and so $f(\mathcal{D})$ becomes pointwise multiplication by the function $f$. That is,

$$
\tilde{\pi}(\hat{f})=\lambda(\hat{f})=f(\mathcal{D}) .
$$

And, hence, if $f$ is a bounded $L^{1}$ function, then:

$$
\operatorname{Tr}(f(\mathcal{D}))=\int_{-\infty}^{\infty} f(t) d t
$$

By this discussion and the previous lemma, we have the following result

Lemma 2.2. If $f \in L^{1}(\mathbf{R}) \cap L^{\infty}(\mathbf{R})$ and $a \in A$ then $\pi(a) f(\mathcal{D})$ is trace-class in $\mathcal{N}$ and

$$
\operatorname{Tr}(\pi(a) f(\mathcal{D}))=\tau(a) \int_{-\infty}^{\infty} f(t) d t
$$

We let $\delta$ be the densely defined (unbounded) $*$-derivation on $A$ which is the infinitesimal generator of the representation $\alpha: \mathbf{R} \rightarrow A u t(A)$ and let $\hat{\delta}$ be the unbounded $*$-derivation on $\mathcal{N}$ which is the infinitesimal generator of the representation $A d \circ \lambda: \mathbf{R} \rightarrow A u t(\mathcal{N})$ (here $A d\left(\lambda_{t}\right)$ denotes conjugation by $\left.\lambda_{t}\right)$. Now if $a \in \operatorname{dom}(\delta)$ then clearly $\pi(a) \in \operatorname{dom}(\hat{\delta})$ and $\pi(\delta(a))=\hat{\delta}(\pi(a))$. One may show that $\pi(\delta(a))$ leaves the domain of $\mathcal{D}$ invariant and

$$
\pi(\delta(a))=2 \pi i[\mathcal{D}, \pi(a)] .
$$

For a discussion of the ideal $\mathcal{L}^{(1, \infty)}$ of compact operators and its role in the semi-finite Dixmier trace we refer to [18].

Theorem 2.3. Let $\tau$ be a faithful finite trace on the unital $C^{*}$-algebra, $A$, which is invariant for an action $\alpha$ of $\mathbf{R}$. Let $\mathcal{N}$ be the semifinite von Neumann algebra $\left(\tilde{\pi}\left(A \times{ }_{\alpha} \mathbf{R}\right)\right)^{\prime \prime}$, and let $\mathcal{D}$ be the infinitesimal generator of the canonical representation $\lambda$ of $\mathbf{R}$ in $U(\mathcal{N})$. Then, the representation $\pi: A \rightarrow \mathcal{N}$ defines a $\mathcal{L}^{(1, \infty)}$ summable semifinite spectral triple $(\mathcal{N}, \mathcal{D})$ for A. Moreover, if $P$ is the nonnegative spectral projection for $\mathcal{D}$ and $u \in U(A)$ is also in the domain of $\delta$, then $T_{u}:=P \pi(u) P$ is $\tau$-Fredholm in $P \mathcal{N} P$ and

$$
\operatorname{ind}\left(T_{u}\right)=\lim _{p \rightarrow 1^{+}} \frac{1}{2}(p-1) \frac{1}{2 \pi i} \operatorname{Tr}\left(\pi\left(u \delta\left(u^{*}\right)\right)\left(1+\mathcal{D}^{2}\right)^{-p / 2}\right)=\frac{1}{2 \pi i} \tau\left(u \delta\left(u^{*}\right)\right) .
$$

By omitting the middle term in $\left({ }^{* *}\right)$ we obtain the statement of Lesch's theorem. The proof of the theorem starts with $\left(^{*}\right)$ in the special case where the endpoints are unitarily equivalent and then deduces the first equality of $(* *)$. The second equality follows from Lemma 2.4. This theorem provides an example of the semi-finite local index formula in the $\mathcal{L}^{(1, \infty)}$ case. This can be seen by comparing $\left({ }^{* *}\right)$ and the $\mathcal{L}^{(1, \infty)}$ case of the local index formula as described in the next Section. 


\section{What IS THE LOCAL INDEX FORMUla IN NCG?}

In the case of odd spectral triples $(\mathcal{A}, \mathcal{H}, \mathcal{D})$ the answer to this question is that the local index formula computes spectral flow from $\mathcal{D}$ to $u \mathcal{D} u^{*}$, where $u \in \mathcal{A}$ is unitary and $[\mathcal{D}, u]$ extends to a bounded operator. As we have seen this is the same as computing the index of the generalised Toeplitz operator $P u P: P \mathcal{H} \rightarrow \mathcal{H}$ where $P=\mathcal{X}_{[0, \infty)}(\mathcal{D})$. The formula is expressed in terms of the residues of zeta functions associated to $\mathcal{D}$ and $u$. In the case of even spectral triples with grading $\Gamma$ it is a formula for the index of $\frac{(1-\Gamma)}{2} q \mathcal{D} q \frac{(1+\Gamma)}{2}$ where $q$ is a projection in $\mathcal{A}$, again in terms of residues of zeta functions associated to $\mathcal{D}$ and $q$. In both cases these zeta function formulas can be seen to be the result of the pairing of a cyclic cocycle (the residue cocycle) with the Chern character in cyclic homology of $u$ or $q$ respectively.

The starting point for the original proof by Connes and Moscovici [25] is the pairing of entire cyclic cohomology and cyclic homology given by the so-called JLO cocycle (Jaffe, Lesniewski and Osterwalder [36]). It seemed natural to the current authors to ask if there might be an analogue of the Connes-Moscovici argument that uses finitely summable techniques only, rather than having to pass through the theta summable JLO formula. Ideally such an argument would also work in semi-finite von Neumann algebras. The main stumbling block for this approach is to find a substitute for the JLO formula.

The germ of the idea of how to do this comes from [22] and [26]. There are hints there that there should be a 'resolvent cocycle' analogous to JLO. Nigel Higson [34] was the first to make this explicit when he developed a new proof of the local index formula using a kind of 'improper resolvent cocycle'.

Higson's paper motivated our introduction in [15] of a genuine 'resolvent cocycle' that leads to the residue cocycle of Connes-Moscovici in a natural fashion. In fact we were able to give a proof of the local index formula starting directly from the spectral flow formula $\left(^{*}\right)$ above. The method is to deduce from $(*)$, in the case where the end point correction terms cancel, a formula for a function valued 'resolvent cocycle' which is, in an appropriate sense, in the $(b, B)$-bicomplex. An advantage of this approach is that it removes a number of side conditions of the original proof, [25], retaining only one that is essential for the statement of the theorem. This condition we call the isolated spectral dimension condition and it replaces the discrete dimension spectrum condition of Connes-Moscovici. Indeed another motivation for the approach of [15] was the concern that, in examples where semi-finite von Neumann algebras arise, it may be difficult to check the discrete dimension spectrum hypothesis. 
Our initial approach to the semi-finite local index formula is contained in the two papers $[15,16]$. The first deals with spectral flow (the odd case) and in the second paper we prove a generalised McKean-Singer formula and then mimic the argument of the odd case to obtain the residue cocycle formula for the even index. Subsequently we found a way to obtain the local index formula directly from the resolvent cocycle without invoking the spectral flow formula $(*)$ (whose proof is not at all easy). This is contained in [17] and incorporates ideas of Higson and Connes-Moscovici on transgression to the Chern character of NCG.

Whichever path is chosen the relative ease of the proof relies on the fact that the resolvent cocycle is not complicated and derives the terms of the residue cocycle in a much simpler fashion than the JLO approach. In addition it works very smoothly in the semifinite von Neumann algebra case.

We will now describe the local index formula and resolvent cocycle for odd spectral triples.

3.1. The odd semi-finite local index formula. First, we require multiindices $\left(k_{1}, \ldots, k_{m}\right), k_{i} \in\{0,1,2, \ldots\}$, whose length $m$ will always be clear from the context. We write $|k|=k_{1}+\cdots+k_{m}$, and define $\alpha(k)$ by

$$
\alpha(k)=1 / k_{1} ! k_{2} ! \cdots k_{m} !\left(k_{1}+1\right)\left(k_{1}+k_{2}+2\right) \cdots(|k|+m) .
$$

With $\sigma_{0,0}=1$, numbers $\sigma_{n, j}$ are defined by the equality

$$
\prod_{j=0}^{n-1}(z+j+1 / 2)=\sum_{j=0}^{n} z^{j} \sigma_{n, j}
$$

These are just the elementary symmetric functions of $1 / 2,3 / 2, \ldots, n-1 / 2$.

If $(\mathcal{A}, \mathcal{H}, \mathcal{D})$ is a smooth semifinite spectral triple (i.e. $\mathcal{A}$ is in the domain of $\delta^{n}$ for all $n$ where $\left.\delta(a)=\left[\left(1+\mathcal{D}^{2}\right)^{1 / 2}, a\right]\right)$ and $T \in \mathcal{N}$, we write $T^{(n)}$ to denote the iterated commutator

$$
\left[\mathcal{D}^{2},\left[\mathcal{D}^{2},\left[\cdots,\left[\mathcal{D}^{2}, T\right] \cdots\right]\right]\right]
$$

where we have $n$ commutators with $\mathcal{D}^{2}$. It follows that operators of the form

$$
T_{1}^{\left(n_{1}\right)} \cdots T_{k}^{\left(n_{k}\right)}\left(1+\mathcal{D}^{2}\right)^{-\left(n_{1}+\cdots+n_{k}\right) / 2}
$$

are in $\mathcal{N}$ when $T_{i}=\left[\mathcal{D}, a_{i}\right]$, or $=a_{i}$ for $a_{i} \in \mathcal{A}$.

Definition If $(\mathcal{A}, \mathcal{H}, \mathcal{D})$ is a smooth semifinite spectral triple, we call

$$
p=\inf \left\{k \in \mathbb{R}: \tau\left(\left(1+\mathcal{D}^{2}\right)^{-k / 2}\right)<\infty\right\}
$$

the spectral dimension of $(\mathcal{A}, \mathcal{H}, \mathcal{D})$. We say that $(\mathcal{A}, \mathcal{H}, \mathcal{D})$ has isolated spectral dimension if for $b$ of the form

$$
b=a_{0}\left[\mathcal{D}, a_{1}\right]^{\left(k_{1}\right)} \cdots\left[\mathcal{D}, a_{m}\right]^{\left(k_{m}\right)}\left(1+\mathcal{D}^{2}\right)^{-m / 2-|k|}
$$


the zeta functions

$$
\zeta_{b}(z-(1-p) / 2)=\tau\left(b\left(1+\mathcal{D}^{2}\right)^{-z+(1-p) / 2}\right)
$$

have analytic continuations to a deleted neighbourhood of $z=(1-p) / 2$.

Now we define, for $(\mathcal{A}, \mathcal{H}, \mathcal{D})$ having isolated spectral dimension and

$$
b=a_{0}\left[\mathcal{D}, a_{1}\right]^{\left(k_{1}\right)} \cdots\left[\mathcal{D}, a_{m}\right]^{\left(k_{m}\right)}\left(1+\mathcal{D}^{2}\right)^{-m / 2-|k|},
$$

the residues

$$
\tau_{j}(b)=\operatorname{res}_{z=(1-p) / 2}(z-(1-p) / 2)^{j} \zeta_{b}(z-(1-p) / 2) .
$$

The hypothesis of isolated spectral dimension is clearly necessary here in order that this definition makes sense. The semifinite local index formula is as follows.

Theorem 3.1. Let $(\mathcal{A}, \mathcal{H}, \mathcal{D})$ be an odd finitely summable smooth spectral triple with spectral dimension $p \geq 1$. Let $N=[p / 2]+1$ where $[\cdot]$ denotes the integer part (so $2 N-1$ is the largest odd integer $\leq p+1$ ), and let $u \in \mathcal{A}$ be unitary. Then if $(\mathcal{A}, \mathcal{H}, \mathcal{D})$ also has isolated spectral dimension then

$s f\left(\mathcal{D}, u^{*} \mathcal{D} u\right)=\frac{1}{\sqrt{2 \pi i}} \sum_{m}^{2 N-1}(-1)^{(m-1) / 2}\left(\frac{(m-1)}{2}\right) ! \phi_{m}\left(u, u^{*}, \ldots, u, u^{*}\right)$

where $\phi_{m}\left(u, u^{*}, \ldots, u, u^{*}\right)$ is

$$
\begin{gathered}
\sum_{|k|=0}^{2 N-1-m} \sum_{j=0}^{|k|+(m-1) / 2}(-1)^{|k|} \alpha(k) \sigma_{(|k|+(m-1)) / 2, j} \\
. \tau_{j}\left(u\left[\mathcal{D}, u^{*}\right]^{\left(k_{1}\right)} \cdots[\mathcal{D}, u]^{\left(k_{m}\right)}\left(1+\mathcal{D}^{2}\right)^{-|k|-m / 2}\right) .
\end{gathered}
$$

When $[p]=2 n$ is even, the term with $m=2 N-1$ is zero, and for $m=$ $1,3, \ldots, 2 N-3$, all the top terms with $|k|=2 N-1-m$ are zero.

The formula represents the evaluation of the semifinite residue cocycle on the Chern character of $u \in \mathcal{A}$.

3.2. The resolvent cocycle. As we explained previously the resolvent cocycle is the natural replacement for the JLO cocycle in the finitely summable situation. It transgresses to the Chern character and is cohomologous (in a sense) to the residue cocycle [17]. As before, we describe only the odd case.

We take $r$ in the right half plane with $\Re(r)$ sufficiently large. Let

$$
R_{s}(\lambda)=\left(\lambda-\left(1+\mathcal{D}^{2}+s^{2}\right)\right)^{-1}
$$

with $s \geq 0$ and $\lambda$ in the line $\{a+i v\}$ where $a \in(0,1 / 2), v \in \mathbf{R}$. For

$$
C(m)=(-\sqrt{2 i}) 2^{m+1} \frac{\Gamma(m / 2+1)}{\Gamma(m+1)}
$$


we define for $m$ odd

$$
\phi_{m}^{r}\left(a_{0}, \ldots, a_{m}\right)=C(m) \int_{0}^{\infty} s^{m}\left\langle a_{0},\left[\mathcal{D}, a_{1}\right], \ldots,\left[\mathcal{D}, a_{m}\right]\right\rangle_{m, s, r} d s
$$

where

$$
\begin{gathered}
\left\langle a_{0},\left[\mathcal{D}, a_{1}\right], \ldots,\left[\mathcal{D}, a_{m}\right]\right\rangle_{m, s, r} \\
=\tau\left(\frac{1}{2 \pi i} \int_{l} \lambda^{-p / 2-r} a_{0} R_{s}(\lambda)\left[\mathcal{D}, a_{1}\right] \cdots\left[\mathcal{D}, a_{m}\right] R_{s}(\lambda) d \lambda\right) .
\end{gathered}
$$

The sequence $\left(\phi_{m}^{r}\right)_{1}^{N}$ is a function valued $(b, B)$ cocycle modulo functions analytic in a half plane containing $r=(1-p) / 2$. We call it the resolvent cocycle. There is an even version as well. The residue cocycle is obtained from the resolvent cocycle by first moving all the resolvents to the right in the integrand of the expression for each $\phi_{m}^{r}$ using a variant on the Connes-Moscovici pseudo-differential calculus. This throws up some remainder terms that are holomorphic at the critical point $(1-p) / 2$. Then one may do the $\lambda$ and $s$ integrals and obtain zeta functions of the kind described in the statement of the local index formula. Then finally one takes residues of the resulting zeta functions at the critical point $r=(1-p) / 2$ on the assumption that they all have an analytic continuation to a neighbourhood of this point (isolated spectral dimension hypothesis). The remainder terms thrown up by the pseudodifferential calculus and the truncation of the resolvent cocycle to finitely many terms are all killed by this process giving an exact expression for the spectral flow in terms of residues of zeta functions.

We remark that in the case of an $\mathcal{L}^{(1, \infty)}$ summable spectral triple the residue cocycle has just one term and this is proportional to the functional on $\mathcal{A} \otimes \mathcal{A}$ given by $\left(a_{0}, a_{1}\right) \mapsto \operatorname{res}_{r=1 / 2} \tau\left(a_{0}\left[\mathcal{D}, a_{1}\right]\left(1+\mathcal{D}^{2}\right)^{-r}\right)$ (cf. the final equation in Theorem 2.5).

Finally, there are applications of semi-finite NCG in various contexts however, we will not discuss them here. Some of these applications are to:

(i) foliations (Connes, Benameur, Fack [6]);

(ii) quantum theory (Connes-Marcolli [28]);

(iii) Cuntz-Krieger systems and $k$-graph $C^{*}$-algebras (Pask-Rennie-Sims [ 43 , 44]);

(iv) Krein's spectral shift function from quantum mechanical scattering theory $[3,4]$.

\section{NEW DIRECTIONS}

Of course not all algebras can be studied by semi-finite NCG. Consider the situation we described in Section 2 in connection with Lesch's formula. There we started with a finite trace on a $C^{*}$-algebra and calculated the spectral flow in an associated semi-finite spectral triple. However this will 
clearly not work if the algebra in question does not admit a trace. This is exactly the situation when type $I I I$ von Neumann algebras arise. Recently two proposals have been made about this, one by Connes-Moscovici [27] and one by us in [14]. Our proposal is predicated on trying to extend, as far as possible, the theory of Toeplitz operators with noncommutative symbol to the situation where the GNS representation associated to a trace on the symbol algebra as in Section 2 is replaced by the GNS representation associated to a KMS state or weight.

We will only consider the situation described in [14] as an application of semifinite NCG in the non-tracial case. In [14] the first of three examples is studied in great detail. This example is that of the Cuntz algebra (which is a type $I I I$ example where there are no non-trivial traces) with its canonical gauge invariant KMS state.

We then discovered that the Cuntz example was not generic in the course of trying to extend our ideas to the case of $S U(2)_{q}$ regarded as a graph $C^{*}$ algebra. We found that $S U_{q}(2)$ violates the 'full spectral subspace' condition which is defined in subsection 4.3 below. For $S U(2)_{q}$, which admits both non-trivial traces and KMS states [20], one may use both the local index formula and the method described in this Section. The information that these yield differs in that the two methods 'see' different parts of the algebra. Both the Cuntz and $S U(2)_{q}$ examples are in turn restrictive as the algebras are unital. Following a suggestion of Matilde Marcolli we then considered, in work in progress, some graphs associated to Mumford curves (a non-unital case).

We remark that the content of [20] is the subject of K. Tong's recent thesis while the final example relies on some joint work of Consani-Marcolli [28, $29,30]$.

4.1. Index for KMS states and weights. After working on the examples summarised above we developed a more systematic point of view in joint work with Marcolli, Neshveyev and Nest. In this subsection we will give an outline of some of this material; see [10] for more of the story.

In previous examples in which spectral flow and the local index formula were used to find invariants of algebras the existence of a trace $[43,18,54]$ was critical. In addition, to construct a spectral triple, the existence of a group action under which the trace was invariant was exploited. In arguing by analogy with this tracial case we were motivated to consider situations where our algebra $A$ admits a KMS state $\tau$ for a strongly continuous circle action $t \rightarrow \sigma_{t}$ of the circle group $\mathbf{T}$. (Or, more generally, we can also work with weights that are KMS for an action of the circle.) Essentially the idea is to look for analogues of Lesch's theorem in the case where one starts with a KMS state. This leads naturally to twisted cyclic theory. 
Let $F$ be the fixed point algebra of $A$ under the circle action and $\Phi$ be the conditional expectation onto the fixed point algebra. We consider circle actions for which the state $\psi$ satisfying the KMS condition is given by taking a trace on $F$ and composing with $\Phi$.

Regard $A$ as a right $F$ module and left $A$-module with a right inner product $(x \mid y)_{R}=\Phi\left(x^{*} y\right)$. Define rank one operators acting on the left by $\Theta_{x, y} z=$ $x(y \mid z)_{R} x, y, z \in A$. Take $\mathcal{N}$ to be the von Neumann algebra generated by these rank one operators in the GNS Hilbert space $H_{\psi}$ of $\psi$. Let $\pi$ be the representation of $A$ acting on the left.

The circle action is implemented by a one parameter group of unitaries $e^{i t \mathcal{D}}, t \in[0,2 \pi]$, acting on $H_{\tau}$. We want to use $\mathcal{D}$ to construct a spectral triple. To simplify notation we will assume that the inverse temperature of our KMS state is $\beta=1$.

We let $\mathcal{M}$ be the fixed point algebra of $A d\left(e^{i t \mathcal{D}}\right)$ in $\mathcal{N}$. This gives a triple of algebras acting on $H_{\tau}$

$$
\mathcal{M} \subset \mathcal{N}, \quad \pi(A)^{\prime \prime} \subset \mathcal{N}, \quad \pi(F) \subset \mathcal{M} \cap \pi(A)^{\prime \prime} .
$$

In examples $\pi(A)^{\prime \prime}$ can be type $I I I$ while we show that $\mathcal{M}$ and $\mathcal{N}$ are always semi-finite von Neumann algebras. We also prove that $\mathcal{D}$ is affiliated to $\mathcal{M}$. We refer to the data $(\mathcal{A}, \mathcal{D}, \mathcal{M})$ as a modular spectral triple.

As usual we want to extract information about the algebras in question by calculating the spectral flow along the path

$$
\left\{\mathcal{D}_{t}=(1-t) \mathcal{D}+t u \mathcal{D} u^{*}\right\}
$$

from $\mathcal{D}$ to $u \mathcal{D} u^{*}$ for unitaries $u$ acting on $H_{\psi}$. This is not well defined in general and we are forced to make some assumptions on the unitaries. Under suitable assumptions our aim is then to try to use our spectral flow formula $\left(^{*}\right)$ to calculate invariants of $A$ using the construction of the auxiliary semifinite algebra $\mathcal{M}$ outlined in the previous paragraphs.

The method we use is to prove from $\left(^{*}\right)$ a residue formula for spectral flow along $\left\{\mathcal{D}_{t}\right\}$ for a class of unitaries $u \in A$ that we term 'modular unitaries'. To introduce this notion we need some further notation. We let $\sigma$ be the non-*-automorphism of $A$ given by setting $t=-i$ in $a \rightarrow \sigma_{t}(a)$. This is the 'twist' in the sense of 'twisted cyclic theory'. Of course $A$ has to be chosen carefully so that this makes sense. The KMS state $\psi$ is a twisted zero cocycle in the sense that $\psi(a b)=\psi(\sigma(b) a)$ for $a, b \in A$. We then define modular unitaries to be those unitaries which satisfy the conditions $u \sigma\left(u^{*}\right) \in F$ and $u^{*} \sigma(u) \in F$. With these conditions, $u \mathcal{D} u^{*}$ is affiliated to $\mathcal{M}$ and $\operatorname{sf}\left(\mathcal{D}, u \mathcal{D} u^{*}\right)$ is well defined in $\mathcal{M}$.

To obtain a finitely summable modular spectral triple we need some further data. In the above situation there is a 'dual trace' coming from the trace 
on the fixed point algebra. It is a faithful normal semifinite trace, that we denote by $\tau$, on $\mathcal{N}$. Unfortunately $\mathcal{D}$ does not satisfy a summability condition with respect to this trace in general (sometimes it does as in the case of $\left.S U_{q}(2)\right)$. To overcome this difficulty we modify the trace by setting $\tau_{\Delta}(T)=\tau\left(e^{-\mathcal{D}} T\right)$ for $T$ in a subalgebra of $\mathcal{N}$. This is known as a 'Gibbs weight'. Then $\tau_{\Delta}$ restricts to a densely defined normal semi-finite trace, say $\phi$, on the fixed point algebra $\mathcal{M}$ of the automorphism group $T \rightarrow e^{i t \mathcal{D}} T e^{-i t \mathcal{D}}$, $T \in \mathcal{N}$. The point of this change of trace, and restriction to the algebra $\mathcal{M}$, is that our modular spectral triple becomes $(1, \infty)$-summable, that is $(1+\mathcal{D})^{-1 / 2} \in \mathcal{L}^{(1, \infty)}(\mathcal{M}, \phi)$.

The conclusion is that, in the setting described in the previous paragraph, the spectral flow formula $\left(^{*}\right)$ enables us to compute, for modular unitaries $u \in A$, the spectral flow $s f_{\phi}\left(\mathcal{D}, u \mathcal{D} u^{*}\right)$ provided we write spectral flow in terms of a residue formula of Connes-Moscovici type. (Note that we still have, in this more general setting, $s f_{\phi}\left(\mathcal{D}, u \mathcal{D} u^{*}\right)=\operatorname{Index}\left(P P^{\prime}\right)$ with $P^{\prime}=$ $u P u^{*} \in \mathcal{M}$.) It is the purpose of the next subsection to explain how to obtain a residue formula from $(*)$.

4.2. The residue formula for modular spectral triples. Let $\mathcal{D}_{a}$ be a self-adjoint densely defined unbounded operator on the Hilbert space $\mathcal{H}$, affiliated to the semifinite von Neumann algebra $\mathcal{M}$. Suppose that for a fixed faithful, normal, semifinite trace $\phi$ on $\mathcal{M}$ we have for $\operatorname{Re}(r)>1 / 2$

$$
\left(1+\mathcal{D}_{a}^{2}\right)^{-r} \in \mathcal{L}^{1}(\mathcal{M}, \phi) .
$$

Let $\mathcal{D}_{b}$ differ from $\mathcal{D}_{a}$ by a bounded self adjoint operator in $\mathcal{M}$. Then for any piecewise $C^{1}$ path $\left\{\mathcal{D}_{t}=\mathcal{D}_{a}+A_{t}\right\}, t \in[a, b]$ in $\mathcal{M}_{0}$ joining $\mathcal{D}_{a}$ and $\mathcal{D}_{b}$, the spectral flow is given by the residue formula [14]:

$$
\begin{gathered}
s f_{\phi}\left(\mathcal{D}_{a}, \mathcal{D}_{b}\right)=\operatorname{Res}_{r=1 / 2} C_{r} s f\left(\mathcal{D}_{a}, \mathcal{D}_{b}\right) \\
=\operatorname{Res}_{r=1 / 2}\left(\int_{a}^{b} \phi\left(\dot{\mathcal{D}}_{t}\left(1+\mathcal{D}_{t}^{2}\right)^{-r}\right) d t+\frac{1}{2}\left(\eta_{\mathcal{D}_{b}}(r)-\eta_{\mathcal{D}_{a}}(r)\right)\right) \\
+\frac{1}{2}\left(\phi\left(P_{\operatorname{ker} \mathcal{D}_{b}}\right)-\phi\left(P_{\operatorname{ker} \mathcal{D}_{a}}\right)\right) .
\end{gathered}
$$

We can consider in this formula, $\mathcal{D}_{b}=u \mathcal{D}_{a} u^{*}$, for $u$ a modular unitary. Then $s f_{\phi}\left(\mathcal{D}_{a}, u \mathcal{D}_{a} u^{*}\right)$ is a homotopy invariant (for homotopies that respect the modular condition). Indeed there is a 'modular $K_{1}$ ' group given by putting an appropriate equivalence relation on modular unitaries although we refer to [14] for this discussion.

Note that we can show that, in certain cases, the residues of the individual terms exist, in particular for the difference of eta terms: $\eta_{\mathcal{D}_{b}}(r)-\eta_{\mathcal{D}_{a}}(r)$. This leads to an interpretation of this combination of eta's as a $b, B$ cochain. 
The above residue formula for $s f_{\phi}\left(\mathcal{D}, u \mathcal{D} u^{*}\right)$ may be rewritten as

$$
\begin{gathered}
\lim _{r \rightarrow 1 / 2}(r-1 / 2)\left(\phi\left(u\left[\mathcal{D}, u^{*}\right]\left(1+\mathcal{D}^{2}\right)^{-r}\right)\right. \\
\left.+\frac{1}{2} \int_{1}^{\infty} \phi\left(\left(\sigma\left(u^{*}\right) u-1\right) \mathcal{D}\left(1+s \mathcal{D}^{2}\right)^{-r}\right) s^{-1 / 2} d s\right) \\
+\frac{1}{2} \phi\left(\left(\sigma\left(u^{*}\right) u-1\right) P_{\operatorname{ker} \mathcal{D}) .}\right.
\end{gathered}
$$

The occurrence of the 'twist' $\sigma$ in equation $(\dagger)$ shows why we cannot eliminate the $\eta$ terms as one would do in the tracial case.

Now assume that the $\eta$ and kernel terms in equation $(\dagger)$ vanish. Then we find that there is a subalgebra of $A$, say $\mathcal{A}$, containing the modular unitaries, such that the functional

$$
\mathcal{A} \otimes \mathcal{A} \ni a_{0} \otimes a_{1} \rightarrow \lim _{r \rightarrow 1 / 2}(r-1 / 2) \phi\left(a_{0}\left[\mathcal{D}, a_{1}\right]\left(1+\mathcal{D}^{2}\right)^{-r}\right)
$$

is a twisted $b, B$-cocycle. In other words the spectral flow is being calculated by a twisted (by $\sigma$ ) residue cocycle when we set $a_{0}=u, a_{1}=u^{*}$. The spectral flow depends only on the modular $K_{1}$ class of $u$.

For some time after discovering this modular $K_{1}$ notion we were not able to fit it into a general framework. In recent work [10] we found a new idea, namely, to study the mapping cone for the inclusion of the fixed point algebra $F$ of the circle action in the algebra $A$. There is a natural $\mathbf{T}$ action on the mapping cone. Basing our approach on ideas of Putnam we show in [10] that there is a map which takes partial isometries satisfying the modular condition into a subgroup of the $\mathbf{T}$-equivariant $K_{1}$-group of the mapping cone. We find that the residue formula for spectral flow may be interpreted as giving a numerical index map on this subgroup. (This suggests the following question: is this a general phenomenon, that is, do twisted cocycles always pair naturally with equivariant K-groups?) These very general results are represented by some examples which we developed with M. Marcolli using graph algebras arising from Mumford curves.

4.3. What the spectral flow formula gives in examples. (i) The Cuntz algebras $O_{n}, n=2,3, \ldots$ are generated by partial isometries $S_{1}, \ldots, S_{n}$ with $S_{i}^{*} S_{j}=\delta_{i j}, \sum_{i} S_{i} S_{i}^{*}=1$. The circle action is given by $\sigma_{t}\left(S_{j}\right)=e^{i t} S_{j}$, $t \in[0,2 \pi]$. In these examples the eta correction terms and kernel terms in equation $(\dagger)$ vanish due to the so-called 'full spectral subspaces condition'. This condition refers to a property of the $k^{t h}$ spectral subspace $A_{k}$ : it is full if,

$$
\overline{A_{k}^{*} A_{k}}=F=\overline{A_{k} A_{k}^{*}} \text {. }
$$

Full spectral subspaces means this condition holds for all integral $k$.

The full spectral subspaces property connects us with our previous discussion. The crossed product $O_{n} \times_{\sigma} \mathbb{T}$ is represented covariantly in $\mathcal{N}$. It was 
observed in [54] that the full spectral subspace condition implies that the crossed product $O_{n} \times{ }_{\sigma} \mathbb{T}$ is faithfully represented in $\mathcal{N}$ and the weak closure of the crossed product is all of $\mathcal{N}$. Hence it is possible to think of this Cuntz example as a precise analogue of the set up for the Lesch result on Toeplitz operators with noncommutative symbol. Now however the symbol is in $O_{n}$ which does not admit a non-trivial trace.

In [14] we associate a modular unitary $u_{\mu, \nu}$ in the $2 \times 2$-matrices over the Cuntz algebra $\mathcal{O}_{n}$ to the product of partial isometries (generators)

$$
S_{\mu} S_{\nu}^{*}=S_{\mu_{1}} \ldots S_{\mu_{m}} S_{\nu_{k}}^{*} \ldots S_{\nu_{1}}^{*} .
$$

Then we obtain

$$
s f_{\phi}\left(\mathcal{D}, u_{\mu, \nu} \mathcal{D} u_{\mu, \nu}^{*}\right)=(m-k)\left(\frac{1}{n^{k}}-\frac{1}{n^{m}}\right) .
$$

We also observed in [14] that, when the eta terms vanish, the residue formula is actually calculating Araki's relative entropy of the two KMS states $\psi$ and $\psi \circ A d u$ of $A$.

(ii) The fermion algebra and the Araki-Woods factors.

Here we use [45]. Fix $\lambda \in(0,1 / 2)$. There is a state on the Fermion algebra (the $C^{*}$-algebra of the canonical anticommutation relations over a separable Hilbert space) that gives rise to the Araki-Woods factor of type $I I I_{\lambda^{\prime}}$ with $\lambda^{\prime}=\lambda /(1-\lambda)[45]$. This state is KMS for the canonical gauge action on the Fermion algebra. It has full spectral subspaces.

From the partial isometry $v$ formed by taking the product of $n$ distinct annihilation operators we obtain a modular unitary $u_{v}$ for which

$$
\begin{gathered}
s f_{\phi}\left(\mathcal{D}, u_{v} \mathcal{D} u_{v}^{*}\right)=n\left((1-\lambda)^{n}-\lambda^{n}\right) \\
=n \frac{\left(e^{n \beta}-1\right)}{\left(1+e^{\beta}\right)^{n}}
\end{gathered}
$$

where $\beta$ is the inverse temperature given by $e^{\beta}=(1-\lambda) / \lambda$. Thus there is a dependence on the inverse temperature and on the number of generators in the product defining the partial isometry. For the fermion algebra this number is the net electric charge. From a physical point of view it is not surprising that the relative entropy should depend on exactly these two parameters.

(iii) $S U_{q}(2)$ viewed as a graph $C^{*}$-algebra.

This example is too complex to discuss fully here and we have to refer the reader to [20]. Thanks to [35] we can view $S U_{q}(2)$ as a graph algebra while at the same time exploiting its Hopf structure in the form of the Haar state. It eventuates that the Haar state is KMS for a certain circle action. However in this case we no longer have full spectral subspaces and consequently the 
'eta cochain' contributes to the spectral flow. This considerably complicates the calculations.

For a product of $m$ partial isometries coming from the graph algebra presentation of $S U_{q}(2)$ we may construct some modular unitaries. On calculating the spectral flow using the residue formula $(\dagger)$ we obtain rational functions of $q$. For example taking a certain product of $m$ generators, we obtain $m\left(1-q^{2}\right)\left(1-q^{2 m}\right)$ for the spectral flow which fits into the pattern above as $q$ is related to the inverse temperature. The $q$ dependence of the modular spectral flow means that this invariant distinguishes algebras $S U(2)_{q}$, $0<q<1$ which are isomorphic as $C^{*}$-algebras but not as Hopf algebras.

In each of these cases one can understand these numerical values once one appreciates from [10] that we are pairing with equivariant mapping cone $K$-theory.

(iv) The example of graph algebras from Mumford curves.

These are generalisations of the $S U_{q}(2)$ situation. One associates to a Mumford curve an infinite directed graph. There is a non-unital graph $C^{*}$-algebra which is associated to this graph. The construction of a modular spectral triple can be carried over to this more general situation. The full spectral subspaces condition fails in general and so we expect that the eta terms will contribute to the spectral flow calculation.

Our method of extending our previous ideas to this more general situation is to introduce the notion of a 'graph weight'. This is a pair of functionals defined respectively on edges and vertices. These define KMS weights on the associated graph $C^{*}$-algebra for certain circle actions when they exist. The spectral flow for partial isometries $v$ constructed from products of the generators of the graph algebra can be calculated but the technicalities of justifying the results are subtle. Specifically we want to calculate, for $v$ a partial isometry given by a product of generators of the graph algebra, the spectral flow $s f\left(\mathcal{D} v v^{*}, v \mathcal{D} v^{*}\right)$ on the subspace $v v^{*} \mathcal{H}$ of the GNS Hilbert space $\mathcal{H}$. A priori this is not defined because we need first to prove that in the spectral flow formula the integrand is in the domain of the trace obtained from our KMS weight.

It was these examples that required a much deeper understanding of spectral flow invariants from weights that are KMS for circle actions than we obtained from the Cuntz and $S U_{q}(2)$ examples and necessitated the more general point of view explained in [10].

There is, however, a pay-off in that we have an interpretation of our results in terms of invariants of Mumford curves. Specifically, to each path in the graph we can associate a product of generators of the graph algebra and 
hence a partial isometry $v$. Using appropriate $v$ 's, $s f\left(\mathcal{D} v v^{*}, v \mathcal{D} v^{*}\right)$ computes a Schottky invariant of the Mumford curve.

(v) How useful is this invariant we are obtaining using 'modular index theory'? There is still further research to be done on the Mumford curves which may shed light on this question. Further insight will of course emerge from the study of other different examples. In work in progress we are studying some crossed product algebras which fit into our general picture with the belief that we will see some finer structure of these algebras using our invariants.

\section{REFERENCES}

[1] M. F. Atiyah, V. K. Patodi, and I. M. Singer, Spectral asymmetry and Riemannian geometry I, Math. Proc. Cambridge Philos. Soc., 77:43-69, 1975.

[2] M. F. Atiyah, V. K. Patodi and I. M. Singer, Spectral asymmetry and Riemannian geometry III, Math. Proc. Cambridge Philos. Soc., 79:71-99, 1976.

[3] N. A. Azamov, A. L. Carey, P. G. Dodds and F. A. Sukochev, Operator integrals, spectral shift and spectral flow, (to appear in Canad. J. Math.), arXiv:math/0703442

[4] N. A. Azamov, A. L. Carey and F. A. Sukochev, The spectral shift function and spectral flow, Commun. Math. Phys., 276:51-91, 2007.

[5] M.-T. Benameur, A. L. Carey, J. Phillips, A. Rennie, F. A. Sukochev and K. P. Wojciechowski, An analytic approach to spectral flow in von Neumann algebras, In Analysis, geometry and topology of elliptic operators, pages 297-352. World Sci. Publ., Hackensack, NJ, 2006.

[6] M.T. Benameur and T. Fack, Type II non-commutative geometry. I. Dixmier trace in von Neumann algebras, Advances in Mathematics 199: 29-87, 2006.

[7] B. Booss-Bavnbek, M. Lesch and J. Phillips, Unbounded Fredholm operators and spectral flow, Canad. J. Math. 57:225-250, 2005.

[8] B. Boo-Bavnbek and K. P. Wojciechowski, Elliptic boundary problems for Dirac operators, Birkhauser, Berlin, 1993.

[9] M. Breuer, Fredholm theories in von Neumann algebras II, Math. Ann., 180:313-325, 1969.

[10] A.L. Carey, S. Neshveyev, R. Nest and A. Rennie, Twisted cyclic theory, equivariant $K K$ theory and KMS States, arXiv:0808.3029.

[11] A. L. Carey and J. Phillips, Unbounded Fredholm modules and spectral flow, Canad. J. Math., 50:673-718, 1998.

[12] A. L. Carey and J. Phillips, Spectral flow in Fredholm modules, eta invariants and the JLO cocycle, K-Theory, 31:135-194, 2004.

[13] A. L. Carey, J. Phillips and A. Rennie, APS Boundary Conditions, $K K$-Theory and Spectral Flow in Graph $C^{*}$-Algebras, arXiv:0711.3028

[14] A. L. Carey, J. Phillips and A. Rennie, Twisted Cyclic Theory and the Modular Index Theory of Cuntz Algebras, arXiv:0801.4605

[15] A. L. Carey, J. Phillips, A. Rennie and F. A. Sukochev, The local index formula in semifinite von Neumann algebras I, Spectral flow. Adv. Math., 202:451-516, 2006.

[16] A. L. Carey, J. Phillips, A. Rennie and F. A. Sukochev, The local index formula in semifinite von Neumann algebras. II. The even case. Adv. Math., 202:517-554, 2006.

[17] A. L. Carey, J. Phillips, A. Rennie and F. A. Sukochev, The Chern character of semifinite spectral triples, Journal of Noncommutative Geometry 2:141-261, 2008

[18] A. L. Carey, J. Phillips, and F. A. Sukochev, On unbounded $p$-summable Fredholm modules, Adv. Math., 151(2):140-163, 2000. 
[19] A. L. Carey, D. S. Potapov and F. A. Sukochev, Spectral flow is the integral of one forms on the Banach manifold of self adjoint Fredholm operators, arXiv:0808.3029

[20] A. L. Carey, A. Rennie and K. Tong, Spectral flow invariants and twisted cyclic theory from the Haar state on $S U_{q}(2)$, arXiv:0802.0317.

[21] A. L. Carey and F. A. Sukochev, Dixmier traces and some applications in noncommutative geometry, UMN, 61:45-110, 2006.

[22] A. Connes, Noncommutative Geometry, Academic Press, 1994.

[23] A. Connes and J. Cuntz, Quasi-homomorphismes, cohomologie cyclique et positivité, Comm. Math. Phys., 114: 515-526, 1988.

[24] A. Connes, M. Marcolli Noncommutative Geometry, Quantum Fields and Motives American Mathematical Society, Providence RI, 2008.

[25] A. Connes and H. Moscovici, The Local Index Formula in Noncommutative Geometry, Geometry and Funct. Analysis, 5:174-243, 1995.

[26] A. Connes and H. Moscovici, Transgression du caractre de Chern et cohomologie cyclique, C. R. Acad. Sci. Paris Sr. I Math. 303:913918, 1986.

[27] A. Connes and H. Moscovici, Type III and spectral triples, arXiv:math/0609703.

[28] C. Consani and M. Marcolli, Noncommutative geometry, dynamics and $\infty$-adic Arakelov geometry, Selecta Math. (N.S.) 10:167-251, 2004.

[29] C. Consani and M. Marcolli, Spectral triples from Mumford curves, International Math. Research Notices, 36:1945-1972, 2003.

[30] C. Consani and M. Marcolli, New perspectives in Arakelov geometry, in "Number theory", 81-102, CRM Proc. Lecture Notes, 36, Amer. Math. Soc., Providence, RI, 2004.

[31] X. Dai and W. Zhang, Higher spectral flow, J. Funct. Anal. 157:432-469, 1958.

[32] T. Fack and H. Kosaki, Generalized s-numbers of $\tau$-measurable operators. Pacific J. Math., 123:269-300, 1986.

[33] E. Getzler, The odd Chern character in cyclic homology and spectral flow. Topology, 32:489-507, 1993.

[34] N. Higson, The local index formula in noncommutative geometry, in Contemporary developments in algebraic K-theory, ICTP Lecture Notes 15, Abdus Salam Int. Cent. Theoret. Phys., Trieste 2003, 447536.

[35] J. H. Hong and W. Szymanski, Quantum spheres and projective spaces as graph Algebras, Commun. Math. Phys. 232:157-188, 2002.

[36] A. Jaffe, A. Lesniewski and K. Osterwalder, Quantum K-Theory I. The Chern Character Commun. Math. Phys. 118:1-14, 1988.

[37] J. Kaad, R. Nest and A. Rennie, $K K$-Theory and Spectral Flow in von Neumann Algebras, math.OA/0701326.

[38] G. G. Kasparov, The Operator $K$-Functor and Extensions of $C^{*}$-Algebras, Math. USSR. Izv. 16:513-572, 1981.

[39] M. Lesch, The uniqueness of the spectral flow on spaces of unbounded self-adjoint Fredholm operators. in Spectral geometry of manifolds with boundary and decomposition of manifolds, B. Boo-Bavnbek, G. Grubb, and K. Wojciechowski, Eds., vol. 366 of Cont. Math. pages 193-224 Amer. Math. Soc., Providence RI, 2005 math.FA/0401411.

[40] M. Lesch, On the Index of the Infinitesimal Generator of a Flow, J. Operator Theory 26:73-92, 1991.

[41] V. Mathai, Spectral flow, eta invariants, and von Neumann algebras, J. Funct. Anal., 109:442-456, 1992.

[42] R. B. Melrose, The Atiyah-Patodi-Singer index theorem, 1993, A K Peters Ltd, Wellesley, USA.

[43] D. Pask and A. Rennie, The Noncommutative Geometry of Graph $C^{*}$-Algebras I: The Index Theorem, J. Funct. Analysis, 233: 92-134, 2006. 
[44] D. Pask, A. Rennie and A. Sims, The noncommutative geometry of k-graph C*_ algebras, J. K-Theory, 1:259-304, 2008.

[45] G. Pedersen, $C^{*}$-Algebras and their Automorphism Groups, London Mathematical Society Monographs, 14, Academic Press, London-New York, 1979.

[46] V. S. Perera, Real valued spectral flow in a type $\mathrm{II}_{\infty}$ factor, Ph.D. Thesis, IUPUI, 1993.

[47] V. S. Perera, Real valued spectral flow in a type $\mathrm{II}_{\infty}$ factor, Houston J. Math., 25:5566, 1999.

[48] J. Phillips, Self-adjoint Fredholm operators and spectral flow, Canad. Math. Bull., 39:460-467, 1996.

[49] J. Phillips, Spectral flow in type I and II factors - a new approach, in Cyclic cohomology and noncommutative geometry (Waterloo, ON, 1995), volume 17 of Fields Inst. Commun., 137-153. Amer. Math. Soc., Providence, RI, 1997.

[50] J. Phillips and I. Raeburn, An Index Theorem for Toeplitz Operators with Noncommutative Symbol Space, J. Funct. Anal., 120:239-263, 1993.

[51] I. Putnam, An excision theorem for the $K$-theory of $C^{*}$-algebras. J. Operator Theory 38:151-171, 1997.

[52] I. M. Singer, Eigenvalues of the Laplacian and invariants of manifolds, in Proceedings of the International Congress of Mathematicians (Vancouver, B. C., 1974), Vol. 1, 187-200. Canad. Math. Congress, Montreal, Que., 1975.

[53] C. Wahl, Spectral flow and winding number in von Neumann algebras, arXiv:0608030.

[54] C. Wahl, Index theory for actions of compact Lie groups on $\mathrm{C}^{*}$-algebras arXiv:0707.3207 